\title{
Combined Impact of the Association between Prepregnancy Body Mass Index and Gestational Weight Gain on the Placental/Umbilical Cord Blood Volume Collected
}

\author{
Atsuko Omori, ${ }^{a}$ Takako Chiba, ${ }^{a}$ Kenji Takahashi, ${ }^{a}$ Kanako Tanaka, ${ }^{b}$ Mami Manabe, ${ }^{b}$ \\ and Ikuo Kashiwakura*,a \\ ${ }^{a}$ Department of Radiological Life Sciences, Division of Medical Life Sciences, Hirosaki University Graduate School of Health Sciences, \\ 66-1 Hon-cho, Hirosaki 036-8564, Japan and ${ }^{b}$ Department of Obstetrics and Gynecology, Hirosaki National Hospital, Tomino-cho, \\ Hirosaki 036-8545, Japan
}

(Received December 18, 2009; Accepted May 16, 2010; Published online May 28, 2010)

\begin{abstract}
Placental/umbilical cord blood (CB) contains multipotent hematopoietic stem/progenitor cells and has been utilized worldwide both clinically and experimentally. Neonatal birth weight is positively correlated with CB volume and with the total number of nucleated cells associated with engraftment and survival after CB transplantation. Considering the recent increase in the frequency of low birth weight $(<2500 \mathrm{~g})$ and the decline in body mass index (BMI) among Japanese women of childbearing age, the present study investigated the combined impact of the prepregnancy BMI and the gestational weight gain on CB volume. From 1998 to 2007, CB samples were obtained from 579 healthy women with singleton vaginal deliveries. The prepregnancy BMI was classified into the underweight, normal, overweight, or obese groups. According to the current gestational weight gain recommendations or other new optimum recommendations, the gestational weight gain was classified into below, within, or above recommendations in each prepregnancy BMI group. The neonatal weight and placental weight had significantly positive effects on the $\mathrm{CB}$ volume. Underweight pregnant women demonstrated significantly lower neonatal and placental weight. According to the current recommendations, no significant difference in the CB volume was observed. According to the new optimum recommendations for underweight pregnant women, a significantly higher $\mathrm{CB}$ volume was obtained from the group within the recommended weight gain range than from the group below the recommended range. In the underweight group, a higher CB volume could be obtained if the upper limit of the gestational weight gain increases by a few kilograms more than the current gestational weight gain recommendations.
\end{abstract}

Key words_— cord blood volume, neonatal weight, prepregnancy body mass index, gestational weight gain

\section{INTRODUCTION}

Placental/umbilical cord blood (CB) contains multipotent hematopoietic stem/progenitor cells. ${ }^{1)}$ $\mathrm{CB}$ can be harvested at no risk to the mother or infant and is increasingly used as an alternative source of hematopoietic stem cell transplantation for patients with diseases such as hematopoietic malignancies. ${ }^{2,3)}$ The total number of nucleated cells infused is a significant factor associated with engraft-

${ }^{*}$ To whom correspondence should be addressed: Department of Radiological Life Sciences, Division of Medical Life Sciences, Hirosaki University Graduate School of Health Sciences, 66-1 Hon-cho, Hirosaki 036-8564, Japan. Tel. \& Fax: +81-172-395938; E-mail: ikashi@cc.hirosaki-u.ac.jp ment and survival after transplantation. ${ }^{4,5)}$ However, it is difficult to predict the number of nucleated cells per CB sample prior to cell processing because of the extremely wide variations in individual samples. Although previous studies have reported the positive correlations between neonatal weight, placental weight, $\mathrm{CB}$ volume, and the total number of nucleated cells, ${ }^{6-8)}$ thus far very little has been elucidated regarding the combined impact of the association between prepregnancy body mass index (BMI) and gestational weight gain on the CB volume collected.

Recently, the increasing number of overweight and obese women has been a major public health problem worldwide, ${ }^{9-11)}$ and maternal obesity is as- 
sociated with a high risk of antenatal/intrapartum complications. ${ }^{12-15)}$ Meanwhile, in Japan a dramatic increase in thinness and a decline in BMI have emerged among Japanese women of childbearing age ${ }^{16)}$ Furthermore, the increasing frequency of low birth-weight infants $(<2500 \mathrm{~g})$ and the decline in the average birth weight have been a nationwide concern for the last few decades. ${ }^{17)}$ Both prepregnancy underweight and low gestational weight gain are associated with low birth weight. ${ }^{18,19)}$

Considering these issues, we hypothesized that gestational weight gain below the recommendations might be one of the factors that negatively affects the $\mathrm{CB}$ volume collected for hematopoietic stem cell transplantation. Over the past decade, we have investigated megakaryocytopoiesis/thrombopoiesis as well as the action of cytokines and their radiation sensitivity using cluster of differentiation 34 positive $\left(\mathrm{CD} 34^{+}\right)$cells prepared from more than $700 \mathrm{CB}$ samples collected for basic research. ${ }^{20-22)}$ These investigations of hematopoietic progenitor cells have shown remarkably significant differences in both the $\mathrm{CB}$ volume and the number of nucleated cells. Moreover, the current criteria of $\mathrm{CB}$ banking are based on the $\mathrm{CB}$ volume and the total number of nucleated cells, and there is no other determining factor of $\mathrm{CB}$ collection from pregnant women who voluntarily offered their CB for donation thus far. Therefore, the present study retrospectively investigated whether weight control during pregnancy could influence the CB volume collected, based on the association between the prepregnancy BMI and gestational weight gain.

\section{MATERIALS AND METHODS}

CB Collection — After the approval by the Committee of Medical Ethics of Hirosaki University Graduate School of Medicine, CB samples were collected at the Fukushi Birth Center located in Goshogawara-shi (Aomori, Japan). Informed consent was obtained from all the mothers prior to collection after an explanation by the midwife during the later gestational period. During the period from August 1998 until the end of September 2007, a total of 579 CB samples were collected from healthy women with singleton vaginal deliveries. Women who had cesarean sections and medical complications of pregnancy were excluded, as relatively healthy pregnant women with no severe diseases tend to give birth at birth centers under the
Medical and Midwifery Law in Japan. According to the guidelines of the Tokyo Cord Blood Bank, $\mathrm{CB}$ was collected after the delivery of the placenta, drained by gravity in an adjacent room, and allowed to flow into a sterile collection bag containing $28 \mathrm{ml}$ of citrate-phosphate-dextrose anticoagulant (CBC20, Nipro, Osaka, Japan) until the flow ceased (approximately $5 \mathrm{~min}$ ). One midwife was primarily responsible for collecting CB samples throughout the entire collection period for this study. Extensive data regarding the medical and family history were voluntarily collected and reported by the staff at the birth center. Of 579 CB samples, a total of 547 were available for analysis due to excluding the CB samples that had either unknown or missing obstetric factor data in the clinical charts.

BMI and Gestational Weight Gain Recommendations - The obstetric factors extracted from the clinical charts were maternal age, paternal age, residential area, maternal occupation, the number of pregnancies, parity, self-reported maternal weight and height, prepregnancy BMI, gestational weight gain, maternal smoking status, gestational age, neonatal gender, duration of labor, neonatal weight and height, placental weight, cord length, and $\mathrm{CB}$ volume ( $\mathrm{CB}$ net weight).

According to the World Health Organization $(\mathrm{WHO})^{23)}$ and the Japan Society for the Study of Obesity, ${ }^{24)}$ prepregnancy BMI $\left(\mathrm{kg} / \mathrm{m}^{2}\right)$ was classified into four groups: underweight $(<18.5)$, normal $(18.5-<25)$, overweight $(25-<30)$, and obese $(\geq 30)$. According to the Ministry of Health, Labor and Welfare of Japan, ${ }^{17)}$ and the Japan Society of Obstetrics and Gynecology, ${ }^{25)}$ the current gestational weight gain recommendations are 9-12 kg for the underweight, $7-12 \mathrm{~kg}$ for normal, and $5-7 \mathrm{~kg}$ for overweight and obese groups. The gestational weight gain in each prepregnancy BMI was classified into three groups: below recommendations, within recommendations, and above recommendations.

Additional Analysis of the Underweight Group — Another set of optimum gestational weight gain recommendations for underweight women has been recently proposed ${ }^{26)}$ after reconsidering the risks related to this group, based on the findings that neonatal weight tends to decrease in the case of gestational weight gain of less than $10 \mathrm{~kg}$ and that heavy for date (HFD) newborns or preeclampsia tend to increase in the case of gestational weight gain of more than $14 \mathrm{~kg}$. Therefore, additional analyses of the underweight group were 
performed according to the new optimum gestational weight gain recommendations, including $10-14 \mathrm{~kg}$ for the underweight, $7-13 \mathrm{~kg}$ for normal, and $<7 \mathrm{~kg}$ for overweight and obese groups.

Statistical Analysis — A univariate analysis was performed using Pearson's correlation coefficient. To determine significant differences among obstetric factors or the $\mathrm{CB}$ volume, a multiple-comparison test was performed using the Bonferroni-Dunn test between each BMI group or between each group of gestational weight gain (below recommendations, within recommendations, and above recommendations) of each BMI group. The statistical analysis was performed using Origin software (Origin Lab, Northampton, MA, U.S.A.) for Windows. A value of $p<0.05$ was considered to be statistically significant.

\section{RESULTS}

Table 1 shows a summary of the characteristics of the $547 \mathrm{CB}$ samples. The mean net weight of the $\mathrm{CB}$ collected was $54.8 \pm 21.6 \mathrm{~g}$. The mean maternal age was $26.6 \pm 4.6$ years, with a range of $17-41$ years. The mean prepregnancy BMI $\left(\mathrm{kg} / \mathrm{m}^{2}\right)$ was $21.2 \pm 3.3$, which was less than the standard BMI of 22. The distribution of prepregnancy BMI was underweight $(<18.5) 17.4 \%$, normal $(18.5-<25)$ $70.4 \%$, overweight $(25-<30) 9.3 \%$, and obese $(\geq 30) 2.9 \%$. The mean neonatal birth weight was $3216.3 \pm 375.3 \mathrm{~g}$, including low birth weight $(<2500 \mathrm{~g}) 1.7 \%$ and macrosomia $(\geq 4000 \mathrm{~g}) 2.9 \%$. The mean gestational age was $39.5 \pm 1.2$ weeks, with a range of 35-43 weeks. Regarding the CB net weight of each BMI group such as underweight $(<18.5)$, normal $(18.5-<25)$, overweight $(25-<30)$, and obese $(\geq 30)$, the data were $54.7 \pm$ $19.9 \mathrm{~g}$ (range $20-237 \mathrm{~g}$ ), $54.1 \pm 21.0 \mathrm{~g}$ (range 10$195.0 \mathrm{~g}$ ), $59.6 \pm 29.2 \mathrm{~g}$ (range $20-210 \mathrm{~g}$ ), and 55.8 $\pm 15.0 \mathrm{~g}$ (range $35.2-80.0 \mathrm{~g}$ ), respectively. Consequently, no significant difference in the $\mathrm{CB}$ net weight was observed between any of the prepregnancy BMI groups.

Table 1. Characteristics of $\mathrm{CB}$ and Obstetric Factors

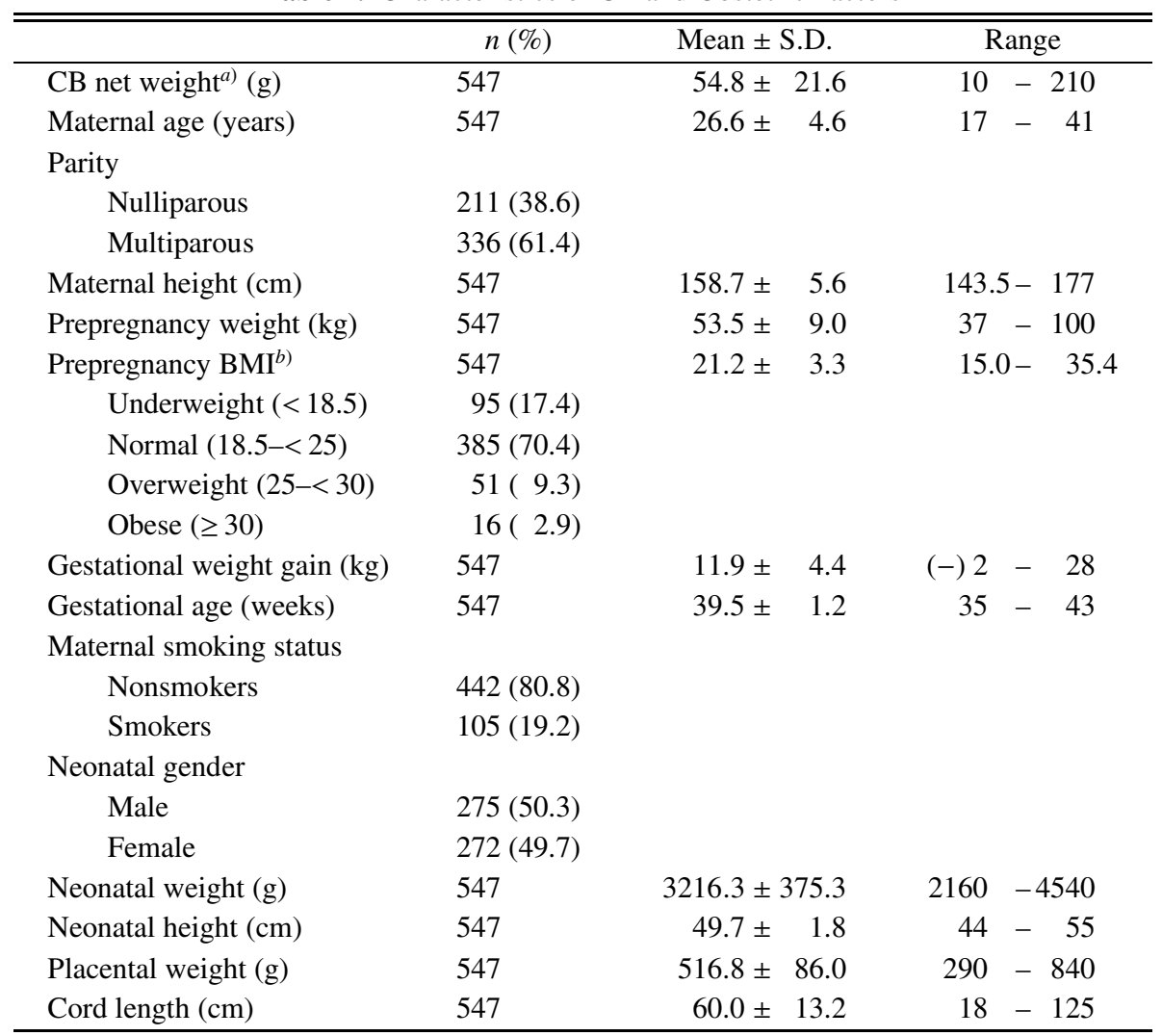

a) $\mathrm{CB}$ net weight indicates $\mathrm{CB}$ volume excluding anticoagulant (CPD). b) BMI: weight in kilograms divided by the square of the height in meters $\left(\mathrm{kg} / \mathrm{m}^{2}\right)$. Prepregnancy BMI was classified into four groups: underweight $(<18.5)$, normal $(18.5-<25)$, overweight $(25-<30)$, and obese $(\geq 30) . n$ : the number of $\mathrm{CB}$ samples. S.D.: standard deviation. Prepregnancy weight was self-reported. 

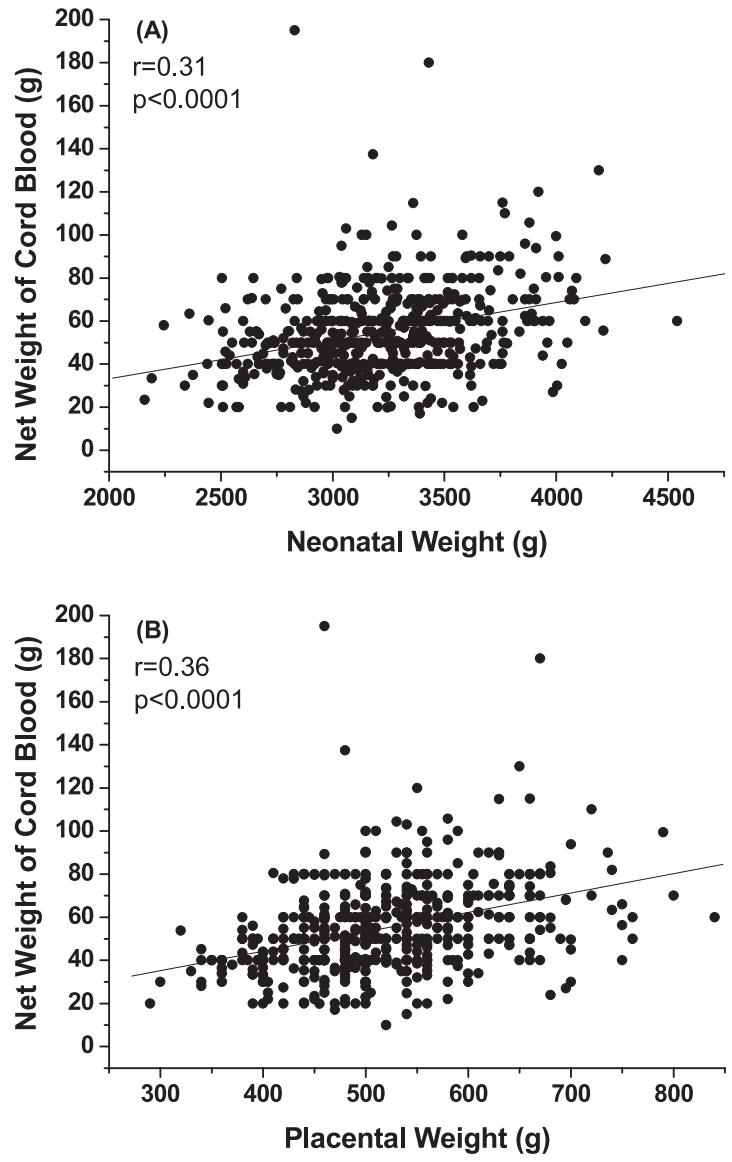

Fig. 1. Correlations between the Obstetric Factors of $\mathrm{CB}$ Samples

Significant positive correlations were observed (A) between neonatal birth weight and CB net weight $(n=547)$, and (B) between placental weight and CB net weight $(n=547)$. Pearson's correlation coefficient: ${ }^{*} p<0.01$.

As shown in Fig. 1, the CB net weight had significantly positive correlations with birth weight and placental weight $(r=0.31, p<0.01 ; r=0.36$, $p<0.01$, respectively). A significantly higher gestational weight gain was observed in the underweight group compared with the other BMI groups (Fig. 2a). In contrast, a significantly lower gestational age, neonatal weight, and placental weight were observed in the underweight group (Fig. 2b, $2 \mathrm{c}$, and 2d).

According to the current gestational weight gain recommendations, no significant difference in the $\mathrm{CB}$ weight was observed between any groups (Fig. 3). On the other hand, according to the new optimum gestational weight gain recommendations $(10-14 \mathrm{~kg})$ recently proposed for the underweight group, a significantly higher CB weight was obtained from the group within the recommended weight gain range $(10-14 \mathrm{~kg})$ compared with the group below the recommended range $(<10 \mathrm{~kg})$, but
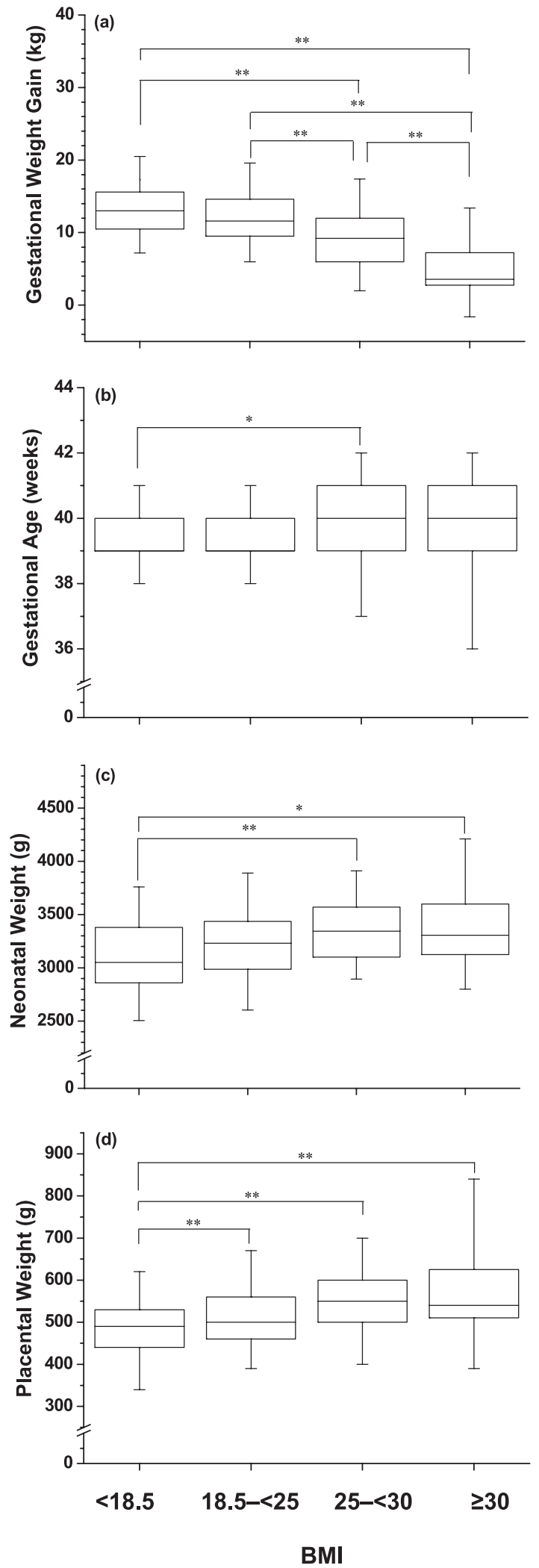

Fig. 2. Multiple-Comparison Test of Obstetric Factors among the Prepregnancy BMI Groups

The obstetric factors are (a) gestational weight gain, (b) gestational age, (c) neonatal weight, and (d) placental weight. Prepregnancy BMI $\left(\mathrm{kg} / \mathrm{m}^{2}\right)$ was classified into four groups: underweight $(<18.5, n=$ 95), normal $(18.5-<25, n=385)$, overweight $(25-<30, n=51)$, and obese $(\geq 30, n=16)$. Bonferroni-Dunn test: ${ }^{*} p<0.05,{ }^{* *} p<0.01$. 

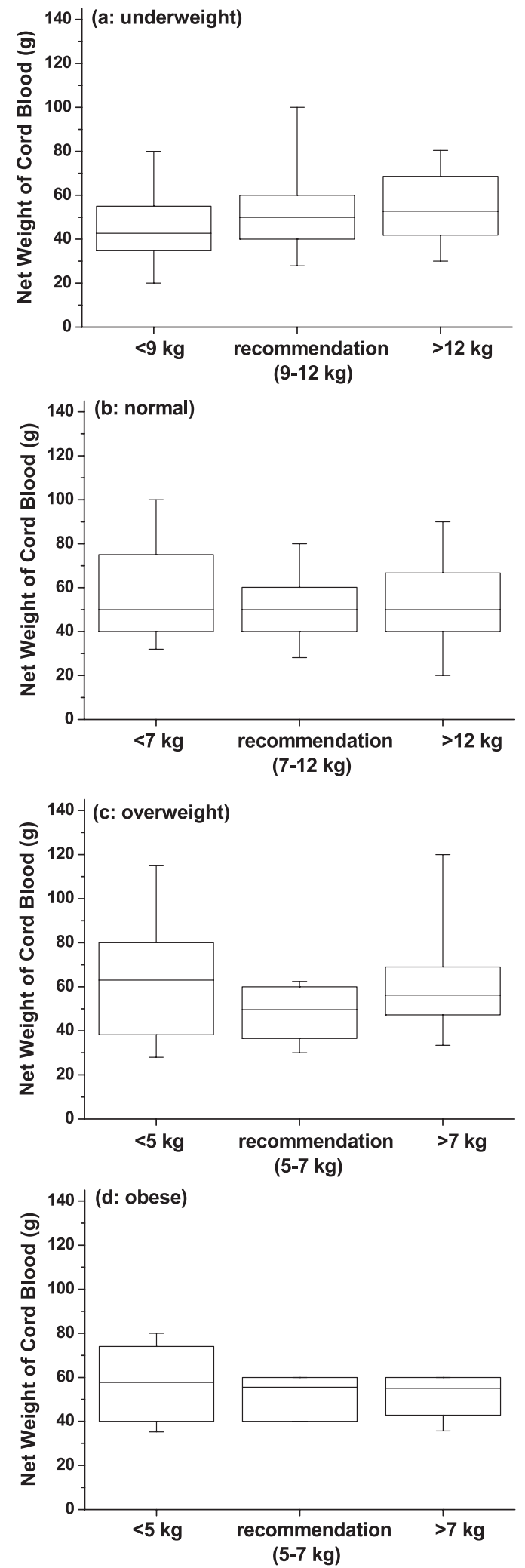

Fig. 3. Multiple-Comparison Test of CB Net Weight According to the Current Gestational Weight Gain Recommendations for Each Prepregnancy BMI

(a: underweight) $<9 \mathrm{~kg}(n=12), 9-12 \mathrm{~kg}(n=31)$, and $>12 \mathrm{~kg}$ ( $n=52)$, (b: normal) $<7 \mathrm{~kg}(n=31), 7-12 \mathrm{~kg}(n=184)$, and $>12 \mathrm{~kg}$ ( $n=170)$, (c: overweight) $<5 \mathrm{~kg}(n=10), 5-7 \mathrm{~kg}(n=7)$, and $>7 \mathrm{~kg}$ $(n=34)$, (d: obese) $<5 \mathrm{~kg}(n=9), 5-7 \mathrm{~kg}(n=3)$ and $>7 \mathrm{~kg}(n=4)$. Bonferroni-Dunn test: no significance between any groups.
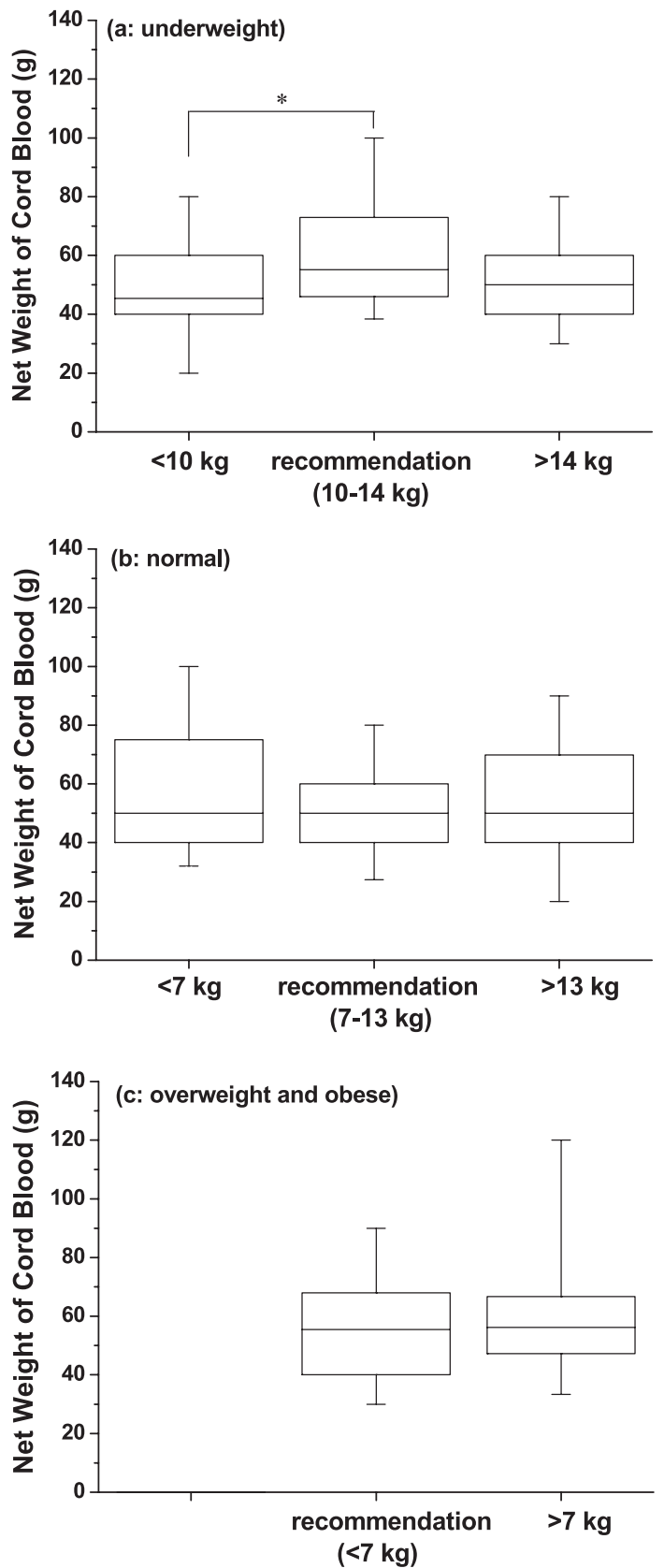

Fig. 4. Multiple-Comparison Test of CB Net Weight According to the New Optimum Gestational Weight Gain Recommendations for Prepregnancy BMI (Underweight)

(a: underweight) $<10 \mathrm{~kg}(n=19), 10-14 \mathrm{~kg}(n=42)$, and $>14 \mathrm{~kg}$ $(n=34)$, (b: normal) $<7 \mathrm{~kg}(n=31), 7-13 \mathrm{~kg}(n=211)$, and $>13 \mathrm{~kg}(n$ $=143)$, (c: overweight and obese) $<7 \mathrm{~kg}(n=29)$ and $>7 \mathrm{~kg}(n=38)$. Bonferroni-Dunn test: ${ }^{*} p<0.05$.

no significant differences were observed between any other groups (Fig. 4). Furthermore, Table 2 illustrates the results of multiple-comparison tests of the $\mathrm{CB}$ net weight, neonatal weight, and placental weight among the recommended weight gain ranges for the underweight group. Despite the higher neonatal weight in the group above recommenda- 
Table 2. Multiple-Comparison Test of the CB Net Weight, Neonatal Weight and Placental Weight According to the New Optimum Gestational Weight Gain Recommendations for the Prepregnancy BMI Underweight Group

\begin{tabular}{lcccc}
\hline \hline $\begin{array}{l}\text { Gestational } \\
\text { weight gain }(\mathrm{kg})\end{array}$ & $n$ & CB net weight $(\mathrm{g})$ & Neonatal weight $(\mathrm{g})$ & Placental weight $(\mathrm{g})$ \\
\hline$<10 \mathrm{~kg}$ & 19 & $46.23 \pm 17.04$ & $2848.95 \pm 291.17$ & $431.83 \pm 57.11$ \\
& & $(45.4)$ & $(2910)$ & $(440)$ \\
$10-14 \mathrm{~kg}$ & 42 & $60.87 \pm 21.81$ & $3135.60 \pm 327.03$ & $504.52 \pm 74.65$ \\
& & $(55.1)^{*}$ & $(3120)$ & $(500)$ \\
$>14 \mathrm{~kg}$ & 34 & $51.90 \pm 16.86$ & $3222.50 \pm 425.22$ & $495.15 \pm 79.22$ \\
& & $(50)$ & $(3250)$ & $(490)$ \\
\hline
\end{tabular}

CB net weight indicates $\mathrm{CB}$ volume excluding anticoagulant (CPD). CB net weight, neonatal weight, and placental weight data indicate the mean \pm standard deviation and (median). Bonferroni-Dunn test: $\mathrm{CB}$ net weight within recommendations $(10-14 \mathrm{~kg}) v s$. below recommendations $(<10 \mathrm{~kg}) .{ }^{*} p<0.05$.

tions (>14 kg), the CB volume was relatively lower in comparison with the group within recommendations $(10-14 \mathrm{~kg})$.

\section{DISCUSSION}

Neonatal birth weight is influenced by obstetric factors, such as prepregnancy BMI, gestational weight gain, gestational age, gravida status, maternal smoking, and maternal age (juvenile or elderly primigravida) ${ }^{27,28)}$ but the placental weight is the strongest determinant of birth weight. ${ }^{29)}$ Various risks are not only associated with maternal obesity but also with underweight women. ${ }^{18,19)}$ Therefore, pregnant women should maintain a normal BMI to achieve a healthy pregnancy outcome. ${ }^{30)}$ In addition, a low weight gain is associated with an increased risk for all of the preterm birth subtypes in comparison with a normal weight gain and low weight gain is an independent and much stronger risk factor for early preterm birth than obesity, although the underlying mechanisms are poorly understood. ${ }^{31)}$

In the present study, neonatal weight and placental weight had significantly positive correlations with the $\mathrm{CB}$ volume, which was consistent with previous reports indicating that bigger is better. $^{6-8)}$ Considering the dramatic increase in thinness among Japanese women of childbearing age, ${ }^{16)}$ along with the decline in the BMI and the average birth weight, ${ }^{17)}$ careful control of the gestational weight gain in underweight women is important for both mothers and neonates. We found that the neonatal weight and placental weight in the underweight group were significantly lower than those in any other BMI group (Fig. 2c and 2d). However, according to the current gestational weight gain rec- ommendations for each BMI group, no significant difference in the $\mathrm{CB}$ volume was observed between any groups (Fig. 3). This could be attributed to relatively healthy women giving birth at birth centers in Japan, thus indicating that a weight gain below, within, or above the recommendations does not seem to have a significant impact on the $C B$ volume in women with uncomplicated pregnancies. However, the detailed mechanisms of the combined impacts of the prepregnancy BMI and gestational weight gain on CB quality still remain unclear. Further studies will be needed to clarify the associations between them.

Interestingly, according to the recently proposed new optimum gestational weight gain recommendations for the underweight group, ${ }^{26)}$ which is slightly higher than the current recommendations for underweight women (10-14 kg vs. 9-12 kg), a higher CB volume was obtained in comparison with the current recommendations $(60.9 \pm 21.8 \mathrm{~g}$ vs. $56.8 \pm 24.1 \mathrm{~g})$. As shown in Table 2, despite the higher neonatal weight in the above recommendations, the CB volume was relatively lower in comparison with the group within recommendations. To some extent, this is probably caused by a lower placental weight in the above recommendations, because no significant differences were observed in smoking status, maternal age, or gestational age between the below recommendations and within the recommendations (data not shown). Consequently, this outcome may be supported by the previous studies reporting that underweight women appeared to benefit from gaining more weight than recommended, ${ }^{32)}$ and that severely thin mothers with very low or very high pregnancy weight gains were at the greatest risk for spontaneous preterm birth. ${ }^{33)}$

There are limitations in the present study due to collecting $\mathrm{CB}$ samples at a birth center. Poten- 
tial confounders such as severe complications could be excluded. Moreover, CB samples were obtained from a regional birth center located in the northern part of Japan. Furthermore, despite the relatively large total number of CB samples, the small group size for underweight pregnant women may have limited the power to detect a difference between groups. However, the well-conducted pregnancy and gestational weight gain controls in each prepregnancy BMI group might strongly reflect the good-quality CB samples collected for donations and possibly lead to a decrease in the low birthweight ratios as well. Regarding the technical factors, even though a working environment may differ from the hospitals affiliated with CB banks, one expert midwife voluntarily performed the CB collection over a decade and her solid experience successfully contributed to consistent, stable CB collection without any problems.

Taken together, in the underweight group, a significantly higher $\mathrm{CB}$ volume could be obtained if the upper limit of the gestational weight gain increases by a few kilograms more than the current gestational weight gain recommendations for underweight pregnant women. Therefore, the upper limit of the gestational weight gain recommendations for underweight pregnant women should be considered as one of the critical factors for $\mathrm{CB}$ collection, providing that a healthy pregnancy is maintained. Our findings may thus give new insight into obstetric factors and provide useful information for improving efficient $\mathrm{CB}$ collection from underweight pregnant women.

Acknowledgements We are indebted to the midwives of the Fukushi Birth Center for collecting the CB samples. This work received support from a Grant for Hirosaki University Institutional Research (2008 and 2009).

\section{REFERENCES}

1) Nakahata, T. and Ogawa, M. (1982) Hemopoietic colony-forming cells in umbilical cord blood with extensive capability to generate mono- and multipotential hemopoietic progenitors. J. Clin. Invest., 70, 1324-1328.

2) O'Brien, T. A., Tiedemann, K. and Vowels, M. R. (2006) No longer a biological waste product: umbilical cord blood. Med. J. Aust., 184, 407-410.

3) Tse, W. and Laughlin, M. J. (2005) Umbilical cord blood transplantation: A new alternative option. Hematology Am. Soc. Hematol. Educ. Program, 377-383.

4) Lim, F., Beckhoven, J., Brand, A., Kluin-Nelemans, J., Hermans, J., Willemze, R., Kanhai, H. and Falkenburg, J. (1999) The number of nucleated cells reflects the hematopoietic content of umbilical cord blood for transplantation. Bone Marrow Transplant., 4, 965-970.

5) George, T. J., Sugrue, M. W., George, S. N. and Wingard, J. R. (2006) Factors associated with parameters of engraftment potential of umbilical cord blood. Transfusion, 46, 1803-1812.

6) Ballen, K. K., Wilson, M., Wuu, J., Ceredona, A. M., Hsieh, C., Stewart, F. M., Popovsky, M. A. and Quesenberry, P. J. (2001) Bigger is better: maternal and neonatal predictors of hematopoietic potential of umbilical cord blood units. Bone Marrow Transplant., 27, 7-14.

7) Nakagawa, R., Watanabe, T., Kawano, Y., Kanai, S., Suzuya, H., Kaneko, M., Watanabe, H., Okamoto, Y., Kuroda, Y., Nakayama, T. and Chugoku-Shikoku Cord Blood Bank (2004) Analysis of maternal and neonatal factors that influence the nucleated and $\mathrm{CD} 4^{+}$cell yield for cord blood banking. Transfusion, 44, 262-267.

8) Mancinelli, F., Tamburini, A., Spagnoli, A., Malerba, C., Suppo, G., Lasorella, R., de Fabritiis, P. and Calugi, A. (2006) Optimizing umbilical cord blood collection: impact of obstetric factors versus quality of cord blood units. Transplant. Proc., 38, 1174-1176.

9) Graves, B. W., DeJoy, S. A., Heath, A. and Pekow, P. (2006) Maternal body mass index, delivery route, and induction of labor in a midwifery caseload. $J$. Midwifery Women's Health, 51, 254-259.

10) Clausen, T., Oyen, N. and Henriksen, T. (2006) Pregnancy complications by overweight and residential area. A prospective study of an urban Norwegian cohort. Acta Obstet. Gynecol. Scand., 85, 526533.

11) Knai, C., Suhrcke, M. and Lobstein, T. (2007) Obesity in Eastern Europe: an overview of its health and economic implications. Econ. Hum. Biol., 5, 392408.

12) Kaiser, P. S. and Kirby, R. S. (2001) Obesity as a risk factor for cesarean in a low-risk population. Obstet. Gynecol., 97, 39-43.

13) Brennand, E. A., Dannenbaum, D. and Willows, N. D. (2005) Pregnancy outcomes of First Nations women in relation to pregravid weight and pregnancy weight gain. J. Obstet. Gynaecol. Can., 27, 936-944. 
14) Abrams, B. and Parker, J. (1988) Overweight and pregnancy complications. Int. J. Obes., 12, 293-303.

15) Callaway, L. K., Prins, J. B., Chang, A. M. and McIntyre, H. D. (2006) The prevalence and impact of overweight and obesity in an Australian obstetric population. Med. J. Aust., 184, 56-59.

16) Takimoto, H., Yoshiike, N., Kaneda, F. and Yoshita, K. (2004) Thinness among young Japanese women. Am. J. Public Health, 94, 1592-1595.

17) Ministry of Health, Labor and Welfare, Japan, The guideline about eating habits for pregnant women, http://www.mhlw.go.jp/index.html (cited 1 February, 2006).

18) Bhattacharya, S., Campbell, D. M., Liston, W. A. and Bhattacharya, S. (2007) Effect of Body Mass Index on pregnancy outcomes in nulliparous women delivering singleton babies. BMC Public Health, 7, 168.

19) Ehrenberg, H. M., Dierker, L., Milluzzi, C. and Mercer, B. M. (2003) Low maternal weight, failure to thrive in pregnancy, and adverse pregnancy outcomes. Am. J. Obstet. Gynecol., 89, 1726-1730.

20) Kashiwakura, I., Murakami, M., Inanami, O., Hayase, Y., Takahashi, T. A., Kuwabara, M. and Takagi, Y. (2002) Effects of amifostine on the proliferation and differentiation of megakaryocytic progenitor cells. Eur. J. Pharmacol., 437, 19-25.

21) Kashiwakura, I., Inanami, O., Takahashi, K., Takahashi, T. A., Kuwabara, M. and Takagi, Y. (2003) Protective effects of thrombopoietin and stem cell factor on $\mathrm{X}$-irradiated $\mathrm{CD} 34^{+}$megakaryocytic progenitor cells from human placental and umbilical cord blood. Radiat. Res., 160, 210-216.

22) Kashiwakura, I., Inanami, O., Abe, Y., Satoh, K., Takahashi, T. A. and Kuwabara, M. (2006) Regenerative induction of megakaryopoiesis and thrombopoiesis in vitro from $\mathrm{X}$-irradiated $\mathrm{CD}^{+} 4^{+}$cells prepared from human placental and umbilical cord blood. Radiat. Res., 166, 345-351.

23) World Health Organization (WHO), Global Database on Body Mass Index, BMI classification, http:// www.who.int/bmi/index.html (cited 1 December, 2009).
24) Japan Society for the Study of Obesity (JASSO), Obesity diagnosis by body weight and BMI criteria, http://wwwsoc.nii.ac.jp/jasso/index.html (cited 1 December, 2009).

25) Japan Society of Obstetrics and Gynecology, Optimum gestational weight gain chart, http://www. jsog.or.jp/index.html (cited 5 November, 2005).

26) Ueda, Y., Maruo, M., Ashitaka, Y., Honda, Y. and Hukayama, T. (2005) Study on relevance of gestational weight gain recommendation implemented at present. Sanpunosinpo, 57, 121-130 (in Japanese).

27) Thame, M., Osmond, C., Bennett, F., Wilks, R. and Forrester, T. (2004) Fetal growth is directly related to maternal anthropometry and placental volume. Eur. J. Clin. Nutr., 58, 894-900.

28) Frederick, I. O., Williams, M. A., Sales, A. E., Martin, D. P. and Killien, M. (2008) Prepregnancy body mass index, gestational weight gain, and other maternal characteristics in relation to infant birth weight. Matern. Child Health J., 12, 557-567.

29) Thame, M., Osmond, C., Wilks, R., Bennett, F. I. and Forrester, T. E. (2001) Second-trimester placental volume and infant size at birth. Obstet. Gynecol., 98, 279-283.

30) Sahu, M. T., Agarwal, A., Das, V. and Pandey, A. (2007) Impact of maternal body mass index on obstetric outcome. J. Obstet. Gynaecol. Res., 33, 655659.

31) Nohr, E. A., Bech, B. H., Vaeth, M., Rasmussen, K. M., Henriksen, T. B. and Olsen, J. (2007) Obesity, gestational weight gain and preterm birth: a study within the Danish National Birth Cohort. Paediatr. Perinat. Epidemiol., 21, 5-14.

32) Rode, L., Hegaard, H. K., Kjaergaard, H., Moller, L. F., Tabor, A. and Ottesen, B. (2007) Association between maternal weight gain and birth weight. $\mathrm{Ob}$ stet. Gynecol., 109, 1309-1315.

33) Salihu, H. M., Mbah, A. K., Alio, A. P., Clayton, H. B. and Lynch, O. (2009) Low pre-pregnancy body mass index and risk of medically indicated versus spontaneous preterm singleton birth. Eur. J. Obstet. Gynecol. Reprod. Biol., 144, 119-123. 